\title{
Psychological well-being and personality in relation to weight loss following behavioral modification intervention in obese women with polycystic ovary syndrome: a randomized controlled trial
}

\author{
Emma Oberg ${ }^{1,2, *}$, Christina Lundell ${ }^{1, *}$, Liselott Blomberg ${ }^{1}$, Sebastian B Gidlöf1,3,4, Petra Tollet Egnell ${ }^{1}$ and \\ Angelica Lindén Hirschberg ${ }^{1,2}$
}

'Department of Women's and Children's Health, Karolinska Institutet, Stockholm, Sweden, ${ }^{2}$ Department of Gynecology and Reproductive Medicine, Karolinska University Hospital, Stockholm, Sweden, ${ }^{3}$ Department of Obstetrics and Gynecology, Stockholm South General Hospital, Stockholm, Sweden, and ${ }^{4}$ Department of Clinical Science, Intervention and Technology (CLINTEC), Unit of Obstetrics and Gynecology, Karolinska Institutet, Stockholm, Sweden

*(E Oberg and C Lundell contributed equally to this work)
Correspondence should be addressed to A L Hirschberg Email angelica.linden-hirschberg@ sll.se

\begin{abstract}
Objective: Little is known about how lifestyle affects psychological well-being in overweight women with polycystic ovary syndrome (PCOS). We investigated the effects of behavioral modification on psychological well-being and the impact of well-being and personality traits on successful weight loss.

Design: A 4-month randomized controlled trial with a 12-month follow-up at a University Hospital.

Methods: Sixty-eight women with PCOS, aged 18 to 40 years with a BMI $\geq 27 \mathrm{~kg} / \mathrm{m}^{2}$, were randomized (1:1) into a behavioral modification program (intervention) or minimal intervention (control). The outcome measures were the psychological well being index and the Swedish universities scales of personality.

Results: At baseline, $60 \%$ had a global psychological well being index corresponding to severe distress and $40 \%$ to moderate distress. There was no significant change in mean global well-being score at 4 months within or between groups. However, after 4 months, the intervention group expressed less anxiety $(P=.035)$, higher general health $(P=.012)$ and lower depressed mood $(P=.033)$. Anxiety and general health tended to differ between groups $(P=.06$, respectively) favoring intervention. In the whole population, women achieving $\geq 5 \%$ weight loss at 12 months ( $n=18$ ) were less anxious at baseline compared to those who had not $(P=.004)$. Personality trait-analysis showed that the weight-loss group had higher social desirability $(P=.033)$ and lower embitterment $(P=.023)$.

Conclusions: Psychological well-being is severely impacted in overweight women with PCOS. Behavioral modification can positively impact dimensions of well-being, although not fully significant, compared to control treatment. Personality factors could contribute to the understanding of successful weight loss.
\end{abstract}




\section{Introduction}

Polycystic ovary syndrome (PCOS) is a common endocrine disorder with a prevalence of $8-13 \%(1,2)$. The most recent international PCOS guidelines recommend the Rotterdam criteria for diagnosis, requiring two out of three of oligo- or anovulation, clinical or biochemical hyperandrogenism and polycystic ovaries on ultrasound, excluding other causes of the symptoms $(3,4)$. PCOS is a heterogeneous condition with several associated features including type 2 diabetes, the metabolic syndrome and abdominal obesity $(5,6)$.

Evidence is emerging that PCOS also has psychological implications $(7,8,9)$. Health-related quality of life, relating to the physical, emotional and social effects of the disorder, is negatively impacted, with weight concerns and infertility most affected $(7,10$, 11). This is important, as psychological well-being is an independent predictor of morbidity and mortality (12). The underlying mechanism for the emotional distress in women with PCOS is unknown; however, androgens and low grade inflammation could contribute (13). A number of studies have shown higher rates of depression and anxiety in women with PCOS compared to the general population independent of body weight $(7,8,14)$ and a shared genetic component between depression and PCOS has been found (15). Furthermore, a large register study showed that women with PCOS have a 50\% increased risk of a psychiatric diagnosis including depression and personality disorders (9).

The most recent international PCOS guidelines recommend screening for impaired quality of life and psychiatric illness (16). Lifestyle intervention - including diet, exercise and behavioral modification - is the firstline treatment in PCOS not only for aspects such as weight concerns, androgen excess and insulin resistance but also for quality of life (16). To our knowledge, only three studies have assessed the efficacy of various lifestyle interventions on emotional well-being in comparison to minimal treatment $(17,18,19)$. A recent Cochrane review concluded that there might be some benefit from lifestyle intervention on quality of life in women with PCOS (20).

Although lifestyle intervention is a first-line treatment in PCOS, achieving substantial weight loss is challenging and further understanding of the factors obstructing success would be beneficial. Attempts have been made to identify patients in other patient populations who may be susceptible to lifestyle intervention for weight-loss purposes. These attempts, involving the assessment of personality prior to intervention, have yielded varying results $(21,22,23,24)$. Personality traits are features which influence mood, thoughts and behavior and are stable over time (21). Personality traits such as impulsivity and sensitivity to reward have been identified as risk factors for weight gain, while self-control and conscientiousness seem protective (25). To our knowledge, the role of personality traits for successful weight loss in overweight women with PCOS has not previously been investigated.

The objective of the present study was twofold. First, to study the efficacy of behavioral modification intervention in improving psychological well-being compared to control treatment. Secondly, to investigate the impact of psychological well-being and personality traits on successful weight loss following behavioral modification intervention.

\section{Subjects and methods}

\section{Study design}

This randomized controlled trial (RCT) was previously described in Oberg et al. (2019) (26). Overweight/ obese women with PCOS were randomized in a 1:1 ratio to receive behavioral modification intervention (intervention) or minimal intervention (control) for 4 months (Fig. 1). Treatment effects within and between groups were evaluated at 4 months. After the initial 4 months, the intervention group received no further intervention, while the control group received the behavioral modification intervention for the following 4 months. Thus, all study participants had undergone the behavioral modification intervention at study completion after 12 months. Differences within the whole group between baseline and 12 months were evaluated. Participants were recruited by newspaper adverts. Consent was obtained from each patient after full explanation of the purpose and nature of all procedures used. The study was approved by the local ethics committee in Stockholm (2012/146-31/3, 2012/1762-32, 2014/1406-32) and registered as a clinical trial (ISRCTN48947168). The primary outcome was weight change, and we have previously published the reproductive outcomes in relation to weight loss (26). Other outcomes include effects on endocrine and metabolic parameters and psychological well-being. This study focuses on the effect of behavioral modification intervention on psychological well-being. 


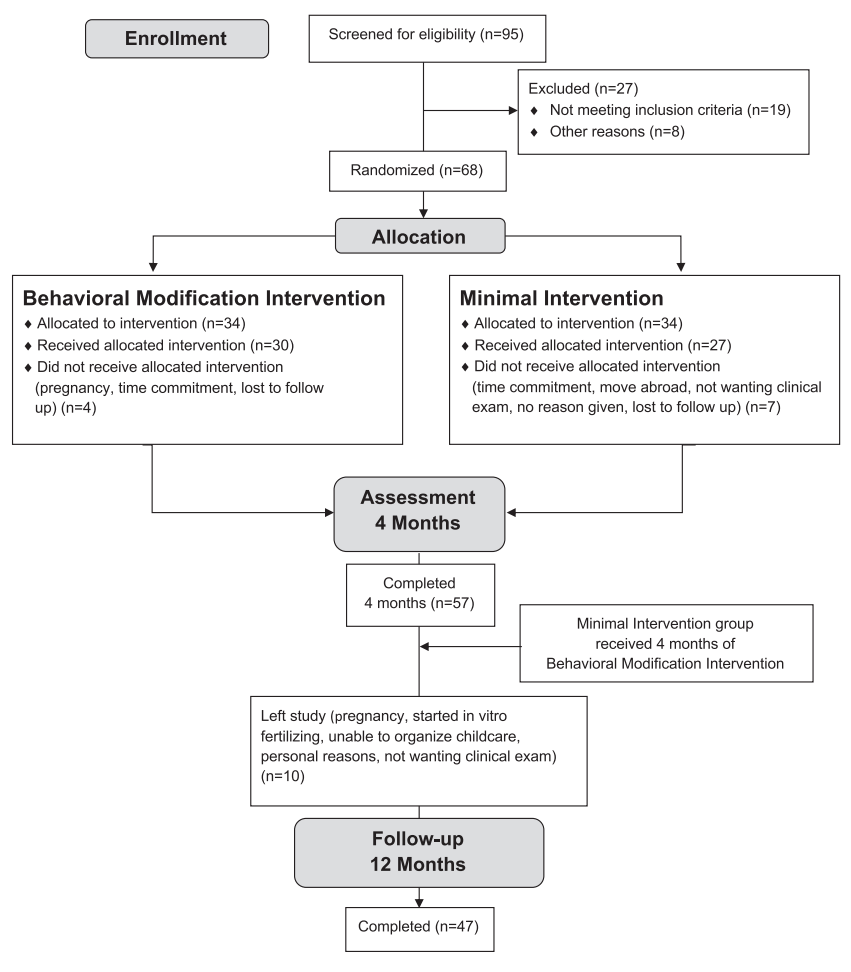

Figure 1

Flow diagram outlining the study design.

\section{Patients}

Study participants had to have a BMI $\geq 27 \mathrm{~kg} / \mathrm{m}^{2}$ and an age between 18-40 years and fulfill all three PCOS diagnostic criteria using the Rotterdam Consensus of oligoor anovulation, clinical or biochemical hyperandrogenism and polycystic ovaries on ultrasound (3). Clinical hyperandrogenism, as manifested by hirsutism, was assessed using the modified Ferriman-Gallwey score, where a score of $\geq 8$ denoted hirsutism. Biochemical hyperandrogenism was defined as a serum testosterone $>310 \mathrm{pg} / \mathrm{mL}$ (27). Polycystic ovaries were defined as at least one ovary having a volume $\geq 10 \mathrm{~mL}$ or $\geq 12$ antral follicles on transvaginal ultrasound $(3,4)$. Exclusion criteria were smoking, other chronic illness, ongoing medication, pregnancy, lactation, having an eating disorder or substantial weight change in the past year. If using a hormonal contraceptive, a washout period of 3 months was used.

\section{Study intervention}

\section{Behavioral modification (Intervention)}

The intervention consisted of a structured training course to achieve long-term weight control (26). Throughout the 4-month intervention, participants attended weekly meetings in small groups led by a lifestyle coach (PTE). Topics discussed included weight control, physical activity, diet, personal leadership, mindfulness, stress management, problem solving, stimulus control and techniques for avoiding instant gratification. The participants prepared for each meeting by reading (28) and personal reflection. Individual coaching sessions were held once monthly to ensure compliance, adapt training and diet regimens and provide personal support.

\section{Minimal intervention (Control)}

The intervention received by the control group was limited to general oral and written information on healthy living, including advice on diet and exercise. This was given by a research midwife and designed to reflect standard care.

\section{Procedures}

Physical examination of all study participants was performed on menstrual cycle day 6-8 at baseline, 4 and 12 months. Women with irregular menstruation or amenorrhea were given $10 \mathrm{mg}$ medroxyprogesterone for 7 days to induce bleeding prior to examination. Height, weight and waist/hip ratio were measured in a standardized manner, a gynecological examination including transvaginal ultrasound was performed and a fasting blood sample was collected to allow for analysis of hormones and binding proteins. Furthermore, the participants filled in the psychological general wellbeing index (PGWBI) questionnaire at baseline, 4 and 12 months and the Swedish universities scales of personality (SSP) at baseline as described subsequently.

\section{Psychological general well-being assessment}

The PGWBI is a validated non-disease specific tool to determine psychological general well-being, and can be used both in clinical practice and research. It assesses the well-being in a patient during the last month (29) and consists of 22 questions, with 6 response choices, ranging from 0 to 5 (12). The questions can be divided into six separate dimensions (scores for each dimension given in brackets): anxiety (0-25), depressed mood (0-15), positive well-being (0-20), self-control (0-15), general health $(0-15)$ and vitality $(0-20)$. All dimensions can be added to achieve a global score (0-110) (29). A larger value reflects greater well-being (e.g. a high score for anxiety indicates a greater well-being). The global PGWBI score can be divided into categories of well-being, where a score between 0 and 
60 indicates severe distress, 60 and 72 moderate distress and 73 and 110 positive well-being (29). Both the scores for the individual dimensions and the global score can be normalized to a scale of $0-100$ to aid the comparison between studies and with other quality of life tools (29). Missing values have been replaced according to the PGWBI user guide (29).

\section{Personality assessment}

SSP is a tool for assessing personality traits in both healthy subjects and psychiatric disease and can be used for research purposes and in clinical practice (30). It is based on a questionnaire with 91 items, each with a choice of four responses that are grouped into 13 personality scales: Mistrust, Trait Irritability, Physical Trait Aggression, Verbal Trait Aggression, Social Desirability, Embitterment, Detachment, Adventure Seeking, Impulsiveness, Lack of Assertiveness, Stress Susceptibility, Psychic Trait Anxiety and Somatic Trait Anxiety (30). The score for each personality scale is used to calculate a T-score, with a mean of 50 and s.D. of 10, based on normative data from a gender stratified Swedish non-patient population (30). It is possible to only use part of the questionnaire if certain personality scales are of interest (30).

\section{Biochemical measurements}

Serum levels of testosterone and other steroids previously reported were determined by liquid chromatography tandem-mass spectrometry (26). Other hormones and binding proteins were analyzed using electrochemiluminescence immunoassay as previously described (26).

\section{Statistical analysis}

SPSS statistical software version 24 (IBM) was used for statistical analysis. In Table 1, categorical data is presented as a percentage. Continuous data is presented either as means \pm S.D. or as means and the $95 \%$ confidence intervals (CI). The difference in the baseline characteristics between the intervention groups was assessed using the Student's $t$-test for continuous data and Fisher's Exact test for categorical data. The effects of treatment within and between groups at 4 months and between baseline and 12 months for the whole group were evaluated using a mixed model (Fig. 2 and Supplementary Tables 1, 2, see section on supplementary materials given at the end of this article). The factors in the model were treatment (behavioral modification intervention and minimal intervention), time (baseline, 4 and 12 months) and the interaction treatment $\mathrm{x}$ time. The results are presented as means and mean differences from baseline together with its 95\% CI. All analyses were carried out on an intention-to-treat (ITT) basis. At 12 months, the population was divided between the ones who managed to lose $\geq 5 \%$ weight (weight-loss group) and the ones who did not (non-weight-loss group). The difference between the two groups was calculated using a mixed model. The difference in distribution of well-being categories between the weight-loss groups was evaluated using Fisher's Exact test. The Spearman rank correlation coefficient was used to measure the association between the PGWBI scores on the one hand and weight loss and different endocrine variables and personality scores on the other. When comparing the mean personality scores with the constant T-score of 50 for a normative population, the difference was considered significant when the CI of the T-score did not include 50. For the association between personality score and successful weight loss, the MannWhitney $U$-test was used to compare the weight loss and the non-weight loss groups. To ensure an ITT approach for these analyses, the last value carried forward was used. $P$-values $<0.05$ were considered statistically significant. The power analysis based on weight change has previously been described and revealed that 30 patients in each group would be sufficient (26).

\section{Results}

\section{Baseline characteristics}

Out of 95 patients screened, 68 fulfilled the entry criteria and were randomized to intervention $(n=34)$ or control $(n=34)$ (Fig. 1) (26). Reasons for exclusion are outlined in Table 1. After randomization, four patients left the intervention group and seven patients the control group, the reasons for which are outlined in Fig. 1, resulting in 30 patients in the intervention group and 27 in the control group ( $84 \%$ in total) completing the initial 4-month intervention period. An additional ten patients left the study before the 12-months follow-up, resulting in 47 patients $(69 \%)$ completing the whole study period (Fig. 1). Table 1 shows the baseline characteristics for the study participants.

\section{Psychological well-being}

Twelve of the 68 study participants (equally distributed between intervention groups) had not filled in sufficient 
Table 1 Baseline characteristics of study participants in the two intervention groups. Data aregiven as mean \pm S.D. or as a proportion (\%) and personality T-scores are given as a mean and 95\% Cl.

\begin{tabular}{|c|c|c|c|}
\hline & $\begin{array}{l}\text { Behavioral modification } \\
\text { intervention }(n=34)\end{array}$ & $\begin{array}{c}\text { Minimal intervention } \\
\qquad(n=34)\end{array}$ & P-value \\
\hline \multicolumn{4}{|l|}{ Demographics\# } \\
\hline Age (year) & $31.0 \pm 5.1$ & $29.9 \pm 5.7$ & .410 \\
\hline Education\# & & & .784 \\
\hline Education - Primary & $0 \%$ & $2.9 \%$ & \\
\hline Education - Secondary & $44.1 \%$ & $35.3 \%$ & \\
\hline Education - University & $55.9 \%$ & $44.1 \%$ & \\
\hline \multicolumn{4}{|l|}{ Marital status\# } \\
\hline In a stable relationship & $79.4 \%$ & $55.9 \%$ & .262 \\
\hline \multicolumn{4}{|l|}{ Anthropometry\# } \\
\hline Body weight (kg) & $92.9 \pm 18.0$ & $93.8 \pm 14.4$ & .807 \\
\hline $\mathrm{BMI}\left(\mathrm{kg} / \mathrm{m}^{2}\right)$ & $33.5 \pm 5.13$ & $34.3 \pm 4.93$ & .514 \\
\hline WHR & $0.9 \pm 0.1$ & $0.9 \pm 0.1$ & .294 \\
\hline Menstrual function\# & & & .590 \\
\hline Regular & 0 & 0 & \\
\hline Oligomenorrhea & $67.6 \%$ & $76.5 \%$ & \\
\hline Amenorrhea & $32.4 \%$ & $23.5 \%$ & \\
\hline \multicolumn{4}{|l|}{ PCO-morphology } \\
\hline Ovarian vol $\geq 10 \mathrm{~mL}(\%)$ & $40.0 \%$ & $30.3 \%$ & .442 \\
\hline No. of follicles $\geq 12(\%)$ & $89.7 \%$ & $87.5 \%$ & 1.00 \\
\hline \multicolumn{4}{|l|}{ Endocrine variables \# } \\
\hline FSH (IU/L) & $6.7 \pm 3.8$ & $5.5 \pm 1.5$ & .105 \\
\hline $\mathrm{LH}(\mathrm{IU} / \mathrm{L})$ & $6.4 \pm 3.4$ & $7.7 \pm 4.5$ & .228 \\
\hline Testosterone (pg/mL) & $358 \pm 110$ & $414 \pm 158$ & .100 \\
\hline $\mathrm{SHBG}(\mathrm{nmol} / \mathrm{L})$ & $27.6 \pm 12.8$ & $26.1 \pm 15.7$ & .660 \\
\hline $\mathrm{AMH}(\mu \mathrm{g} / \mathrm{L})$ & $7.3 \pm 5.2$ & $7.2 \pm 6.2$ & .985 \\
\hline \multicolumn{4}{|l|}{ Hyperandrogenism } \\
\hline Biochemical hyperandrogenisma & $71.9 \%$ & $70.0 \%$ & 1.00 \\
\hline mFG score & $10.5 \pm 6.9$ & $11.5 \pm 7.9$ & .578 \\
\hline $\mathrm{mFG} \geq 8(\%)$ & $61 . \overline{3} \%$ & $59.4 \%$ & 1.00 \\
\hline \multicolumn{4}{|c|}{ Psychological general well being index score ${ }^{b}$} \\
\hline Global PGWBI (0-110) & $56.6 \pm 6.4$ & $58.3 \pm 4.9$ & .299 \\
\hline Anxiety $(0-25)$ & $15.7 \pm 3.2$ & $15.7 \pm 2.8$ & .921 \\
\hline Depressed mood (0-15) & $9.1 \pm 1.8$ & $9.4 \pm 1.3$ & .508 \\
\hline Positive well-being (0-20) & $10.4 \pm 1.9$ & $10.9 \pm 1.7$ & .347 \\
\hline Self control (0-15) & $4.3 \pm 1.8$ & $5.2 \pm 1.7$ & .075 \\
\hline General health $(0-15)$ & $7.1 \pm 1.7$ & $8.1 \pm 2.4$ & .622 \\
\hline Vitality $(0-20)$ & $9.0 \pm 2.8$ & $9.0 \pm 2.0$ & .949 \\
\hline \multicolumn{4}{|l|}{ Personality (T-score $)^{c}$} \\
\hline Somatic trait anxiety & 53.3 (49.2 to 57.4$)$ & $55.0(50.5$ to 59.6$)$ & .561 \\
\hline Psychic trait anxiety & 54.3 (49.9 to 58.7 ) & 53.4 (48.5 to 58.2$)$ & .761 \\
\hline Stress susceptibility & 55.2 (50.3 to 60.2$)$ & 51.3 (45.7 to 56.8 ) & .284 \\
\hline Lack of assertiveness & 51.6 (47.8 to 55.4$)$ & $52.0(46.7$ to 57.3$)$ & .897 \\
\hline Impulsiveness & 53.8 (49.8 to 57.7$)$ & 54.1 (48.7 to 59.4$)$ & .909 \\
\hline Adventure seeking & 51.5 (47.2 to 55.8$)$ & $52.7(49.1$ to 56.2$)$ & .679 \\
\hline Detachment & 51.4 (47.1 to 55.8$)$ & 47.8 (43.3 to 52.3$)$ & .240 \\
\hline Embitterment & 53.0 (49.3 to 56.8$)$ & 57.2 (51.6 to 62.7$)$ & .192 \\
\hline Social desirability & 51.0 (46.3 to 55.7$)$ & $53.7(49.3$ to 58.1$)$ & .407 \\
\hline Verbal trait aggression & 50.4 (45.8 to 54.9$)$ & 45.7 (40.8 to 50.6$)$ & .157 \\
\hline Physical trait aggression & 47.2 (43.6 to 50.7$)$ & 48.6 (44.7 to 52.5$)$ & .576 \\
\hline Trait irritability & 55.7 (50.7 to 60.7$)$ & 53.3 (48.4 to 58.1$)$ & .487 \\
\hline Mistrust & 53.2 (47.9 to 58.6$)$ & 56.5 (51.3 to 61.7$)$ & .382 \\
\hline
\end{tabular}

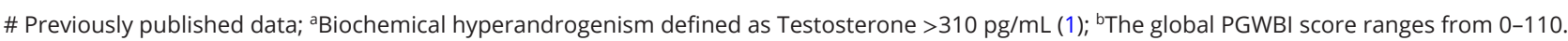
The numerical range for each PGWBI dimension is given in brackets. A higher score indicates a higher well-being; 'The Personality T-score is calculated based on a normative score of 50 for a gender stratified Swedish non-patient population.

$\mathrm{AMH}$, anti müllerian hormone; FSH, follicle stimulating hormone; LH, luteinizing hormone; mFG, modified Ferriman-Gallwey; PGWBI, psychological general well being index; SHBG, sex hormone-binding globulin; WHR, waist/hip ratio. 


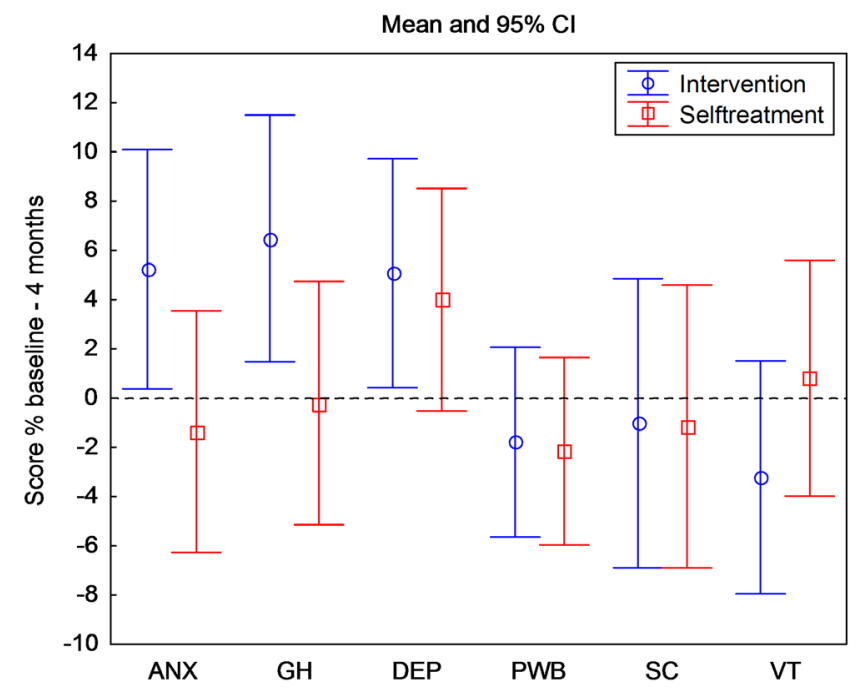

Figure 2

Normalized PGWBI change at 4 months in percent from baseline for each dimension split into intervention $(n=23)$ and self treatment (control) $(n=26)$. Values given are mean and $95 \% \mathrm{Cl}$ calculated using a mixed model. A larger value reflects greater psychological well-being (e.g. a high score for anxiety indicates greater well-being). ANX, anxiety; GH, general health; DEP, depressed mood; PWB, positive well-being; SC, self control; VT, vitality.

PGWBI data, leaving 56 women for inclusion in the final analysis.

At baseline, 60\% of all study participants had a global PGWBI score corresponding to severe distress and $40 \%$ to moderate distress. No patients at baseline fell into the category of positive well-being. There was no difference between the intervention groups $(P=.375)$.

There was no significant change in the normalized mean global PGWBI score between baseline and 4 months for the intervention group $(1.5,95 \% \mathrm{CI}$ : -0.8 to 3.7$)$ and the control group ( $-0.2,95 \% \mathrm{CI}:-2.5$ to 2.0$)$ and no significant difference between groups $(1.7,95 \% \mathrm{CI}$ : -1.5 to $4.9, P=.298)$. There was, however, a significant improvement of the normalized mean score in three of the individual PGWBI dimensions for the intervention group at 4 months: anxiety 5.2 (95\% CI: 0.4 to $10.1, P=.035)$, general health 6.5 (95\% CI: 1.5 to $11.5, P=.012)$ and depressed mood 5.1 (95\% CI: 0.4 to $9.7, P=.033$ ) (Fig. 2). The change in anxiety and general health tended to be significantly different between the two groups $(P=.06$, respectively, Supplementary Table 1).

Between baseline and 12 months, there was no significant change in the proportion of women falling into each category of well-being. Furthermore, there was

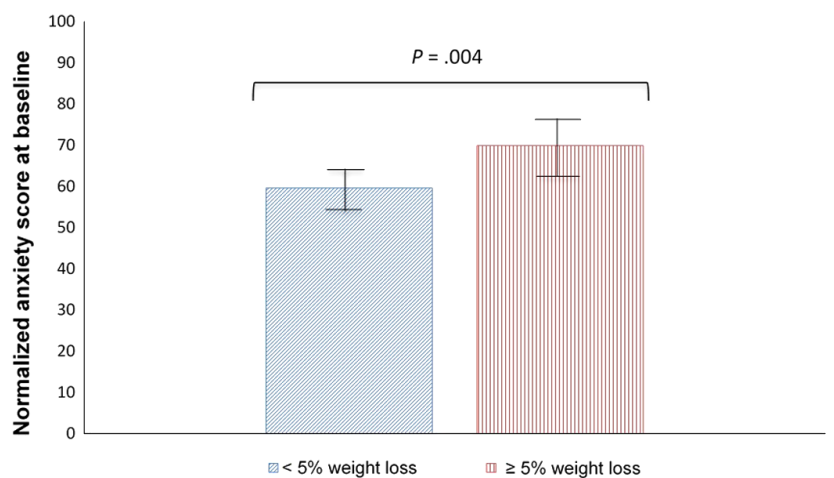

\section{Figure 3}

Normalized anxiety scores at baseline in the weight-loss group $(n=16)$ and non-weight loss group $(n=33)$ at the 12 months follow-up. The significance level was calculated using a mixed model. Analysis done on an ITT basis.

no significant difference in mean global PGWBI score or in any of the dimensions (Supplementary Table 2). However, the subgroup of women managing to lose $\geq 5 \%$ weight (weight-loss group, $n=18$ of all study participants) at 12 months had a significantly higher normalized anxiety score (i.e. less anxiety) at baseline (69.6, 95\% CI: 64.2 to 75.1$)$ compared to the non-weight loss group (59.6, 95\% CI: 55.8 to 63.5), $P=.004$ (Fig. 3). In addition, at 12 months, there was a larger proportion of women in the category of moderate distress vs the category of severe distress in the weight-loss group $(P=.005)$ (Fig. 4).

\section{Personality}

Of the total study population $(n=68)$, SSP questionnaires were missing for 19 women resulting in a total $n=57$ for personality assessment. Table 1 shows the mean baseline personality T-scores and 95\% CI for the intervention and control groups. Figure 5 shows the deviation of the mean T-scores from the normative personality T-scores of 50 for the whole study population categorized into the weightloss group and the non-weight loss group. At baseline, the weight-loss group had a significantly lower Embitterment score $(P=.023)$ and higher Social Desirability score $(P=.033)$ than the non-weight loss group (Fig. 5 and Supplementary Table 3). In the non-weight loss group, the personality T-scores at baseline indicated that they exhibited more Somatic Trait Anxiety 55.5 (95\% CI: 51.6 to 59.4), Psychic Trait Anxiety 55.5 (95\% CI: 51.3 to 59.6), Stress Susceptibility 55.6 (95\% CI: 50.7 to 60.5 ), Impulsiveness 55.9 (95\% CI: 52.2 to 59.6), Embitterment 56.5 (95\% CI: 53.0 to 60.0), Trait Irritability 56.2 (95\% CI: 


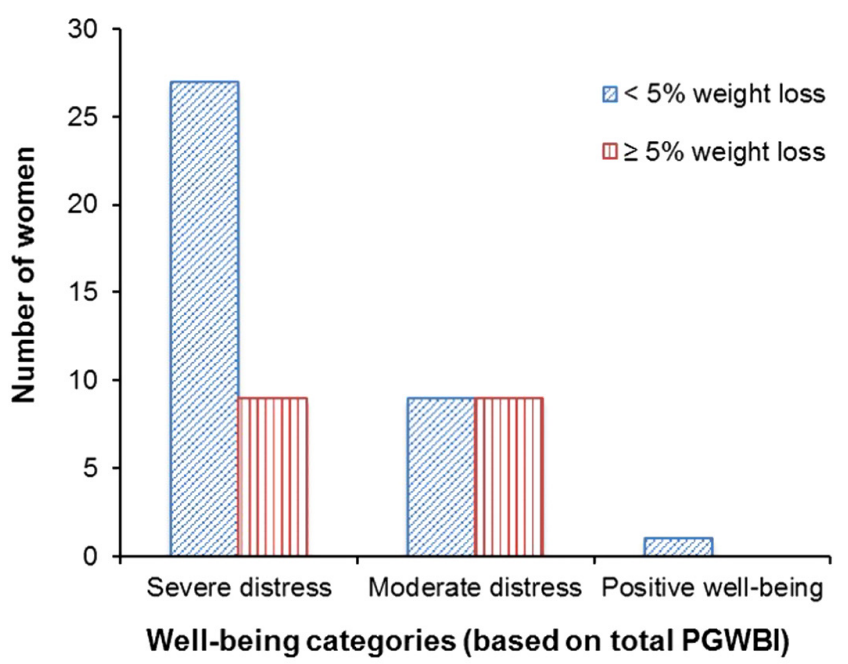

\section{Figure 4}

Number of study participants in each category of well-being at 12 months for the subgroup of women who achieved $\geq 5 \%$ weight loss and the ones who did not lose weight. The overall distribution of well-being categories were significantly different between the two subgroups, $P=.005$, using Fisher's Exact test.

51.4 to 61.0) and Mistrust 55.7 (95\% CI: 50.8 to 60.6) than a normative female population (Supplementary Table $3)$. On the other hand, the weight-loss group expressed more Social Desirability 56.1 (95\% CI: 52.3 to 59.9) and less Physical Trait Aggression 45.7 (95\% CI: 41.7 to 49.7) than a normative female population (Supplementary Table 3). We found no statistically significant correlations

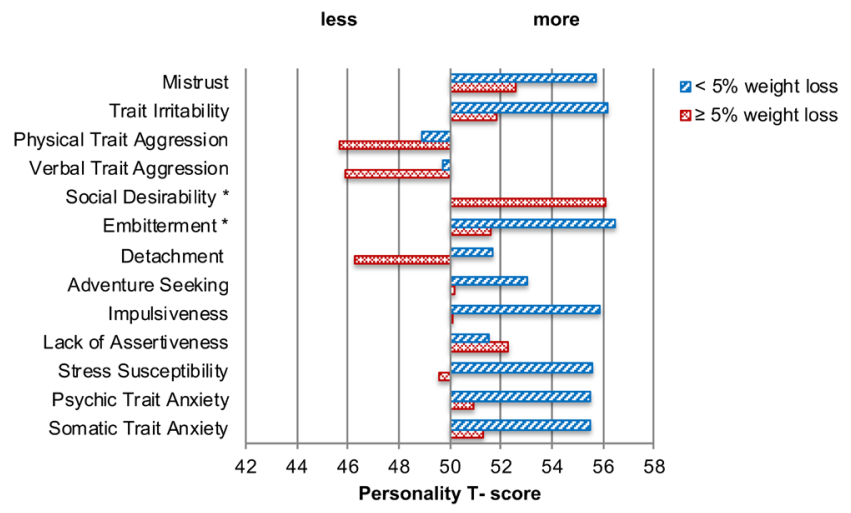

\section{Figure 5}

Deviation from normative Personality T-score (50) for the subgroup of women who achieved $\geq 5 \%$ weight loss ( $n=17$ ) and the ones who did not lose weight $(n=32)$. * indicates significance between the two subgroups $(P<0.05)$, using the Mann-Whitney U-test. Analysis done on an ITT basis. between the mean global PGWBI score and the individual personality traits.

\section{Discussion}

To our knowledge, this is the first study investigating psychological well-being and personality traits following behavioral modification intervention compared to minimal intervention in women with PCOS. In this RCT, we found that psychological well-being in overweight/ obese women with PCOS in general is poor. Lifestyle intervention improved some aspects of well-being such as anxiety, general health and depressed mood, albeit this improvement was not fully significant when compared to controls. In addition, we showed the importance of well-being for achieving weight loss and that certain personality traits at baseline (lower embitterment and higher social desirability) were associated with successful weight loss.

We were surprised by the very low level of psychological well-being at baseline. In fact, no study participant was in the category of positive well-being, in which $70 \%$ of a normal population is found (29). Previous work has similarly shown that the health-related quality of life in women with PCOS is considerably lower than in non-PCOS women $(10,11,31,32,33)$. When comparing the psychological well-being of our PCOS population with PGWBI scores for other medical conditions, our group scored substantially lower $(29,34,35)$. This agrees with Coffey et al. (2006), who found their PCOS population scoring markedly lower in all dimensions of healthrelated quality of life (using the non-disease specific SF-36 tool) than patients with, for example, diabetes, arthritis and heart disease (31).

To our knowledge, the effect of lifestyle intervention in comparison to minimal intervention on well-being has only been investigated by a few studies, with varying results $(17,18,19)$. This was summarized in a recent Cochrane review (20). More specifically, Stener-Victorin et al. (2013) assessed the effects of physical exercise compared to controls and found improvements to some domains of general health-related quality of life (Short Form (SF) - 36: physical functioning) and to the infertility aspect of the disease-specific PCOS quality of life questionnaire (PCOSQ) (17). Stefanaki et al. (2015) studied the effects of a mindfulness stress management program on disease-specific quality of life compared to controls and found no difference between the groups following intervention (18). A small pilot study by Vizza et al. (2016) 
found positive effects on both disease-specific (PCOSQ: emotions, weight and infertility problems) and general health-related quality of life domains (SF-36: physical functioning, vitality, social functioning, role emotional and mental health) following progressive resistance training compared to controls (19). Based on these results, the Cochrane review concluded that lifestyle intervention may improve quality of life, particularly the dimensions of emotions and infertility in the PCOSQ (20). However, the effect was uncertain due to high heterogeneity, large variability and wide confidence intervals (20). We found a tendency of improvement in the dimensions of anxiety and general health following lifestyle intervention in comparison to minimal intervention. Furthermore, there was a significant within-group difference in some dimensions of well-being following intervention, which is in line with previous studies using different assessment tools $(17,18,36,37)$. In contrast to the PCOSQ, the PGWBI is a non-disease specific, validated instrument for measuring psychological well-being, but not physical functions, which can be used for comparison with normative data and other medical conditions (29).

We and others have previously shown that achieving weight loss in this population of overweight/obese women with PCOS is challenging $(26,38,39,40)$. Lifestyle intervention is the recommended first-line treatment and it is therefore important to identify ways of selecting patients likely to benefit from intervention $(16,20)$. We found that a higher well-being at baseline was associated with successful weight loss. Similar results have been found by Dokras et al. (2016) and Moran et al. (2006) (33, 41).

In this study, we also found a relationship between successful weight loss and some personality traits. The only other study we found that uses SSP to assess personality in relation to weight loss is by Elfhag et al. (2005) looking at eating behavior in both male and female obese patients (23). They found that higher Adventure Seeking was related to lower BMI, and higher Embitterment, Trait Irritability, Mistrust, Detachment and Lack of Assertiveness were related to uncontrolled eating. Furthermore, hunger was positively related to Lack of Assertiveness (23). However, another study using the Temperament and Character Inventory (TCI) tool for assessing personality found low scores of novelty seeking in a non-PCOS female population to be significantly associated with weight loss following medical and cognitive behavioral interventions (22). Similarly, Sullivan et al. (2007), using the TCI, found a link between high novelty seeking scores and decreased success in achieving weight loss following behavioral intervention (24).
We found a small number of studies of personality in women with PCOS, all of which found this group of women to be more likely to have a personality disorder and to have significantly different scores in personality scales compared to controls $(9,42)$. However, to our knowledge, ours is the first study investigating personality traits in relation to behavioral modification intervention in women with PCOS. We found significantly higher personality scores for social desirability and lower for embitterment in the weight-loss group compared to the non-weight-loss group. Furthermore, personality scores for the non-weight loss group were significantly higher than the normative scores for several personality traits (Somatic Trait Anxiety, Psychic Trait Anxiety, Stress Susceptibility, Impulsiveness, Embitterment, Trait Irritability and Mistrust). For the weight-loss group, there were only two personality traits that differed from the normative score (higher Social Desirability and lower Physical Trait Aggression). Although personality traits appear to be of importance for managing to lose weight, our results need to be confirmed by other studies.

This study has several strengths: one is the randomized design, allowing for comparison with controls at 4 months and a follow-up at 12 months. The fact that all women eventually received the intervention helped with recruitment and provided a long-term follow-up of the whole study population. Another strength is the PGWBItool used, which assesses psychological well-being and allows for comparison with normative values and other patient populations. However, a limitation is that we have not related the well-being to PCOS specific symptoms as is done in the PCOSQ-tool. In this study, we chose to relate psychological well-being to weight changes, as other authors have highlighted weight concerns as being of major importance for quality of life in these women (7). In a previously published paper from our trial, we demonstrated improvements to endocrine parameters, menstrual function and fertility following behavioral modification, which also could have impacted the results in this paper (26).

A relatively small sample size $(n=68$, Fig. 1$)$ could explain only finding a tendency toward significance between groups, whereas there were significant withingroup changes following intervention. Furthermore, as one of our exclusion criteria for participation was taking medication, the well-being could have been negatively affected at 12 months, as the women received no treatment for their PCOS symptoms. However, only six patients had to stop their hormonal contraceptive prior to joining the 
study. The drop-out rate (16\% after 4 months and $31 \%$ after one year) is a potential limitation of the study but is, in fact, lower than, or on a par with, similar studies (19, $36,37,43)$. One explanation for the drop-out rate is the high level of commitment needed to participate. Another important point is that our study population fulfilled all three PCOS diagnostic criteria. The results might therefore not be applicable to a more general PCOS population of different phenotypes (3).

We conclude that psychological well-being is severely impacted in this patient group and that behavior modification can have a positive effect on some dimensions of well-being, although not fully significant compared to control treatment. Managing to achieve weight loss is associated with less anxiety and higher well-being at baseline, highlighting the importance of assessing and addressing the psychological status prior to embarking on a lifestyle-changing program. Furthermore, despite extensive and time-consuming efforts to achieve weight loss through behavioral modification, the intervention only seems to be effective in a subgroup of women. Personality factors could contribute to the understanding of the success of lifestyle treatment.

\section{Supplementary materials}

This is linked to the online version of the paper at https://doi.org/10.1530/ EJE-20-0066.

\section{Declaration of interest}

The authors declare that there is no conflict of interest that could be perceived as prejudicing the impartiality of this study.

\section{Funding}

This work was supported by the Swedish Research Council (A L H 201702051), the Stockholm County Council (A L H 2016-0349) and Karolinska Institutet $(A \mathrm{~L} H)$.

\section{Acknowledgements}

The authors would like to thank Berit Legerstam and Siv Rödin Andersson at the Women's Health Research Unit at the Karolinska University Hospital for their logistical support as well as the statistician Elisabeth Berg at Karolinska Institutet for help with the statistical analysis.

\section{References}

1 March WA, Moore VM, Willson KJ, Phillips DI, Norman RJ \& Davies MJ. The prevalence of polycystic ovary syndrome in a community sample assessed under contrasting diagnostic criteria. Human Reproduction 201025 544-551. (https://doi.org/10.1093/ humrep/dep399)

2 Bozdag G, Mumusoglu S, Zengin D, Karabulut E \& Yildiz BO. The prevalence and phenotypic features of polycystic ovary syndrome: a systematic review and meta-analysis. Human Reproduction 201631 2841-2855. (https://doi.org/10.1093/ humrep/dew218)

3 Rotterdam EA-SPCWG. Revised 2003 consensus on diagnostic criteria and long-term health risks related to polycystic ovary syndrome. Fertility and Sterility 200481 19-25.

4 Teede H, Misso ML, Costello MF, Dokras A, Laven J, Moran L, Piltonen T \& Norman RJ. International Evidence-Based Guidelines for the Assessment and Managment of Polycystic Ovary Syndrome 2018. Monash University: Melbourne Australia, 2018.

5 Diamanti-Kandarakis E \& Dunaif A. Insulin resistance and the polycystic ovary syndrome revisited: an update on mechanisms and implications. Endocrine Reviews 201233 981-1030. (https://doi. org/10.1210/er.2011-1034)

6 Moran LJ, Misso ML, Wild RA \& Norman RJ. Impaired glucose tolerance, type 2 diabetes and metabolic syndrome in polycystic ovary syndrome: a systematic review and meta-analysis. Human Reproduction Update 201016 347-363. (https://doi.org/10.1093/ humupd/dmq001)

7 Dokras A, Stener-Victorin E, Yildiz BO, Li R, Ottey S, Shah D, Epperson N, Teede H. Androgen Excess-Polycystic Ovary Syndrome Society: position statement on depression, anxiety, quality of life, and eating disorders in polycystic ovary syndrome. Fertility and Sterility 2018109 888-899. (https://doi.org/10.1016/j. fertnstert.2018.01.038)

8 Cooney LG, Lee I, Sammel MD \& Dokras A. High prevalence of moderate and severe depressive and anxiety symptoms in polycystic ovary syndrome: a systematic review and meta-analysis. Human Reproduction 201732 1075-1091. (https://doi.org/10.1093/humrep/ dex044)

9 Cesta CE, Mansson M, Palm C, Lichtenstein P, Iliadou AN \& Landen M. Polycystic ovary syndrome and psychiatric disorders: co-morbidity and heritability in a nationwide Swedish cohort. Psychoneuroendocrinology 201673 196-203. (https://doi. org/10.1016/j.psyneuen.2016.08.005)

10 Bazarganipour F, Taghavi SA, Montazeri A, Ahmadi F, Chaman R \& Khosravi A. The impact of polycystic ovary syndrome on the healthrelated quality of life: a systematic review and meta-analysis. Iranian Journal of Reproductive Medicine 201513 61-70.

11 Barnard L, Ferriday D, Guenther N, Strauss B, Balen AH \& Dye L. Quality of life and psychological well being in polycystic ovary syndrome. Human Reproduction 200722 2279-2286. (https://doi. org/10.1093/humrep/dem108)

12 Lundgren-Nilsson Å, Jonsdottir IH, Ahlborg Jr G \& Tennant A. Construct validity of the psychological general well being index (PGWBI) in a sample of patients undergoing treatment for stressrelated exhaustion: a Rasch analysis. Health and Quality of Life Outcomes 201311 2. (https://doi.org/10.1186/1477-7525-11-2)

13 Veltman-Verhulst SM, Boivin J, Eijkemans MJ \& Fauser BJ. Emotional distress is a common risk in women with polycystic ovary syndrome: a systematic review and meta-analysis of 28 studies. Human Reproduction Update 201218 638-651. (https://doi.org/10.1093/ humupd/dms029)

14 Brutocao C, Zaiem F, Alsawas M, Morrow AS, Murad MH \& Javed A. Psychiatric disorders in women with polycystic ovary syndrome: a systematic review and meta-analysis. Endocrine 201862 318-325. (https://doi.org/10.1007/s12020-018-1692-3)

15 F, Karaderi T, Jones MR, Meun C, He C, Drong A, Kraft P, Lin N, Huang $\mathrm{H}$, Broer L et al. Large-scale genome-wide meta-analysis of polycystic ovary syndrome suggests shared genetic architecture for different diagnosis criteria. PLoS Genetics 201814 e1007813. (https:// doi.org/10.1371/journal.pgen.1007813)

16 Teede HJ, Misso ML, Costello MF, Dokras A, Laven J, Moran L, Piltonen T, Norman RJ \& International PCOS Network. Recommendations from the international evidence-based guideline 
for the assessment and management of polycystic ovary syndrome. Fertility and Sterility 2018110 364-379. (https://doi.org/10.1016/j. fertnstert.2018.05.004)

17 Stener-Victorin E, Holm G, Janson PO, Gustafson D \& Waern M. Acupuncture and physical exercise for affective symptoms and health-related quality of life in polycystic ovary syndrome: secondary analysis from a randomized controlled trial. BMC Complementary and Alternative Medicine 201313 131. (https://doi.org/10.1186/14726882-13-131)

18 Stefanaki C, Bacopoulou F, Livadas S, Kandaraki A, Karachalios A, Chrousos GP \& Diamanti-Kandarakis E. Impact of a mindfulness stress management program on stress, anxiety, depression and quality of life in women with polycystic ovary syndrome: a randomized controlled trial. Stress 201518 57-66. (https://doi.org/10 $.3109 / 10253890.2014 .974030)$

19 Vizza L, Smith CA, Swaraj S, Agho K \& Cheema BS. The feasibility of progressive resistance training in women with polycystic ovary syndrome: a pilot randomized controlled trial. BMC Sports Science, Medicine and Rehabilitation 20168 14. (https://doi.org/10.1186/ s13102-016-0039-8)

20 Lim SS, Hutchison SK, Van Ryswyk E, Norman RJ, Teede HJ \& Moran LJ. Lifestyle changes in women with polycystic ovary syndrome. Cochrane Database of Systematic Reviews 20193 CD007506. (https://doi.org/10.1002/14651858.CD007506.pub4)

21 De Panfilis C, Torre M, Cero S, Salvatore P, Dall'Aglio E, Marchesi C, Cabrino C, Aprile S \& Maggini C. Personality and attrition from behavioral weight-loss treatment for obesity. General Hospital Psychiatry 200830 515-520. (https://doi.org/10.1016/j. genhosppsych.2008.06.003)

22 Dalle Grave R, Calugi S, Compare A, El Ghoch M, Petroni ML, Colombari S, Minniti A \& Marchesini G. Personality, attrition and weight loss in treatment seeking women with obesity. Clinical Obesity $20155266-272$.

23 Elfhag K. Personality correlates of obese eating behaviour: Swedish universities scales of personality and the three factor eating questionnaire. Eating and Weight Disorders 200510 210-215. (https:// doi.org/10.1007/BF03327487)

24 Sullivan S, Cloninger CR, Przybeck TR \& Klein S. Personality characteristics in obesity and relationship with successful weight loss. International Journal of Obesity 200731 669-674. (https://doi. org/10.1038/sj.ijo.0803464)

25 Gerlach G, Herpertz S \& Loeber S. Personality traits and obesity: a systematic review. Obesity Reviews 201516 32-63. (https://doi. org/10.1111/obr.12235)

26 Oberg E, Gidlof S, Jakson I, Mitsell M, Tollet Egnell P \& Hirschberg AL. Improved menstrual function in obese women with polycystic ovary syndrome after behavioural modification intervention-a randomized controlled trial. Clinical Endocrinology 201990 468-478. (https://doi.org/10.1111/cen.13919)

27 Labrie F, Cusan L, Gomez JL, Cote I, Berube R, Belanger P, Martel C $\&$ Labrie C. Effect of intravaginal DHEA on serum DHEA and eleven of its metabolites in postmenopausal women. Journal of Steroid Biochemistry and Molecular Biology 2008111 178-194. (https://doi. org/10.1016/j.jsbmb.2008.06.003)

28 Ingvar M \& Eldh G. Hjärnkoll på vikten. Natur and Kultur 2010.

29 Chassany O, Dimenas E, Dubois D, Wu A \& Dupuy H The Psychological General Well-Being Index (PGWBI) User Manual. Lyon, France: MAPI Research Institute, 2004.

30 Gustavsson JP, Bergman H, Edman G, Ekselius L, von Knorring L \& Linder J. Swedish universities scales of personality (SSP): construction, internal consistency and normative data. Acta Psychiatrica Scandinavica 2000102 217-225. (https://doi. org/10.1034/j.1600-0447.2000.102003217.x)
31 Coffey S, Bano G \& Mason HD. Health-related quality of life in women with polycystic ovary syndrome: a comparison with the general population using the Polycystic Ovary Syndrome Questionnaire (PCOSQ) and the Short Form-36 (SF36). Gynecological Endocrinology 200622 80-86. (https://doi. org/10.1080/09513590600604541)

32 Ching HL, Burke V \& Stuckey BG. Quality of life and psychological morbidity in women with polycystic ovary syndrome: body mass index, age and the provision of patient information are significant modifiers. Clinical Endocrinology 200766 373-379. (https://doi. org/10.1111/j.1365-2265.2007.02742.x)

33 Dokras A, Sarwer DB, Allison KC, Milman L, Kris-Etherton PM, Kunselman AR, Stetter CM, Williams NI, Gnatuk CL, Estes SJ et al. Weight loss and lowering androgens predict improvements in health-related quality of life in women with PCOS. Journal of Clinical Endocrinology and Metabolism 2016101 2966-2974. (https://doi. org/10.1210/jc.2016-1896)

34 Petta CA, Ferriani RA, Abrao MS, Hassan D, Rosa E Silva JC, Podgaec S \& Bahamondes L. Randomized clinical trial of a levonorgestrelreleasing intrauterine system and a depot GnRH analogue for the treatment of chronic pelvic pain in women with endometriosis. Human Reproduction 200520 1993-1998. (https://doi.org/10.1093/ humrep/deh869)

35 Isidoro SI, Salvaggio A, Lo Bue A, Romano S, Marrone O \& Insalaco G. Effect of obstructive sleep apnea diagnosis on health related quality of life. Health and Quality of Life Outcomes 20151368. (https://doi.org/10.1186/s12955-015-0253-1)

36 Thomson RL, Buckley JD, Lim SS, Noakes M, Clifton PM, Norman RJ $\&$ Brinkworth GD. Lifestyle management improves quality of life and depression in overweight and obese women with polycystic ovary syndrome. Fertility and Sterility 201094 1812-1816. (https://doi. org/10.1016/j.fertnstert.2009.11.001)

37 De Frene V, Verhofstadt L, Lammertyn J, Stuyver I, Buysse A \& De Sutter P. Quality of life and body mass index in overweight adult women with polycystic ovary syndrome during a lifestyle modification program. Journal of Obstetric, Gynecologic, and Neonatal Nursing 201544 587-599. (https://doi.org/10.1111/15526909.12739)

38 Hoeger K, Davidson K, Kochman L, Cherry T, Kopin L \& Guzick DS. The impact of metformin, oral contraceptives, and lifestyle modification on polycystic ovary syndrome in obese adolescent women in two randomized, placebo-controlled clinical trials. Journal of Clinical Endocrinology and Metabolism 200893 4299-4306. (https:// doi.org/10.1210/jc.2008-0461)

39 Turan V, Mutlu EK, Solmaz U, Ekin A, Tosun O, Tosun G, Mat E, Gezer C \& Malkoc M. Benefits of short-term structured exercise in non-overweight women with polycystic ovary syndrome: a prospective randomized controlled study. Journal of Physical Therapy Science 201527 2293-2297. (https://doi.org/10.1589/ jpts.27.2293)

40 Palomba S, Giallauria F, Falbo A, Russo T, Oppedisano R, Tolino A, Colao A, Vigorito C, Zullo F \& Orio F. Structured exercise training programme versus hypocaloric hyperproteic diet in obese polycystic ovary syndrome patients with anovulatory infertility: a 24-week pilot study. Human Reproduction 200823 642-650. (https://doi. org/10.1093/humrep/dem391)

41 Moran LJ, Noakes M, Clifton PM, Wittert GA, Williams G \& Norman RJ. Short-term meal replacements followed by dietary macronutrient restriction enhance weight loss in polycystic ovary syndrome. American Journal of Clinical Nutrition 200684 77-87. (https://doi.org/10.1093/ajcn/84.1.77)

42 Scaruffi E, Franzoi IG, Civilotti C, Guglielmucci F, La Marca L, Tomelini M, Veglia F \& Granieri A. Body image, personality profiles 
and alexithymia in patients with polycystic ovary syndrome (PCOS). Journal of Psychosomatic Obstetrics and Gynecology 2018 1-10.

43 Jedel E, Labrie F, Oden A, Holm G, Nilsson L, Janson PO, Lind AK, Ohlsson C \& Stener-Victorin E. Impact of electro-acupuncture and physical exercise on hyperandrogenism and oligo/amenorrhea in women with polycystic ovary syndrome: a randomized controlled trial. American Journal of Physiology: Endocrinology and Metabolism 2011300 E37-E45. (https://doi.org/10.1152/ajpendo.00495.2010)

Received 23 January 2020

Revised version received 5 April 2020

Accepted 24 April 2020 